\title{
A Concise Microwave-Assisted Synthesis of 2-Aminoimidazole Marine Sponge Alkaloids of the Isonaamines Series
}

\author{
D. S. Ermolat'ev, ${ }^{a}$ V. L. Alifanov, ${ }^{b}$ V. B. Rybakov, ${ }^{b}$ E. V. Babaev, ${ }^{* b}$ E. V. Van der Eycken*a \\ a Laboratory for Organic and Microwave-Assisted Chemistry (LOMAC), University of Leuven, \\ Celestijnenlaan 200F, 3001 Leuven, Belgium \\ Fax +32(16)327990; E-mail: erik.vandereycken@chem.kuleuven.be \\ b Department of Chemistry, Moscow State University, 119991 Moscow, Russia \\ Fax +7(495)9393929; E-mail: babaev@ org.chem.msu.ru \\ Received 31 March 2008; revised 9 April 2008
}

\begin{abstract}
A short and efficient route to 1,4-substituted 2-aminoimidazole alkaloids starting from the easily accessible 2-alkylaminopyrimidines and $\alpha$-bromo aldehydes is reported. The formation of the intermediate imidazo[1,2-a]pyrimidinium salts and subsequent cleavage were facilitated by microwave irradiation. Marine sponge alkaloids preclathridines $\mathrm{A}, \mathrm{C}$ and isonaamines $\mathrm{A}, \mathrm{C}, \mathrm{D}$ were obtained in high yields using the optimized one-pot two-step procedure.
\end{abstract}

Key words: natural products, ring opening, cleavage, imidazo[1,2a]pyrimidin-1-ium salts, 2-aminoimidazoles

Marine sponges have been proven to be a source of biologically active alkaloids and their metabolites. Among the calcareous sponges, the genera Leucetta and Clathrina are a rich source of imidazole alkaloids. Since the first discovery of 2-aminoimidazole alkaloids in marine sponges by Kashman's group in $1987,{ }^{1}$ a number of preclathridine and isonaamine alkaloids, representing a family of 1,4substituted 2-aminoimidazoles $\mathbf{1}$ bearing one or two substituted benzyl moieties, has been isolated and synthesized in the last two decades (Table 1). ${ }^{2}$ Many 2aminoimidazole alkaloids have been reported to have cytotoxic, antimicrobial, and antifungal properties. ${ }^{3}$
The reported synthetic approaches to 1,4-dialkyl-2-aminoimidazoles $\mathbf{1}$ include quite a lengthy iminophosphorane-mediated synthesis from $\alpha$-azido esters, ${ }^{4}$ the condensation of poorly available $\alpha$-amino ketones with cyanamide, ${ }^{5,6}$ or a multistep derivatization of the protected imidazole core. ${ }^{7,8}$

We have recently communicated a facile one-pot two-step procedure for the synthesis of diversely substituted 2-aminoimidazoles from $\alpha$-bromocarbonyl compounds and substituted 2-aminopyrimidines. ${ }^{11}$ This methodology could serve as a novel, practical, and general approach to marine alkaloids of the family $\mathbf{1}$ (Table 1 ).

Here, we report a short and efficient total synthesis of preclathridine and isonaamine alkaloids $1 \mathbf{a}-\mathbf{e}$ based on the condensation of 2-aminopyrimidines $\mathbf{3}$ and $\alpha$-bromo aldehydes $\mathbf{4}$ and subsequent cleavage of the intermediate imidazopyrimidinium salts 2 (Scheme 1). Although heterocyclization reactions of $\alpha$-bromo aldehydes are hardly known due to their high reactivity, in our preliminary studies we were able to synthesize several 1,4-disubstituted 2-aminoimidazoles in high yields applying a onepot two-step microwave-assisted protocol. ${ }^{11}$

Table 1 1,4-Substituted 2-Aminoimidazole Marine Sponge Alkaloids<smiles>[R]c1cc(Cc2cn(P)c(N)n2)ccc1[2H]</smiles>

\begin{tabular}{|c|c|c|c|c|c|}
\hline Alkaloid & $\mathrm{R}^{1}$ & $\mathrm{R}^{2}$ & $\mathrm{R}^{3}$ & Isolated & Synthesis \\
\hline Preclathridine A (1c) & $\mathrm{Me}$ & $-\mathrm{OCH}_{2} \mathrm{O}-$ & & $1992^{9}$ & $1996,{ }^{7} 1999^{4}$ \\
\hline Preclathridine C (1i) & $\mathrm{Me}$ & $\mathrm{H}$ & $\mathrm{OH}$ & $1991^{5}$ & $1991,,^{5} 1999^{4}$ \\
\hline Isonaamine A (1j) & 4-Hydroxybenzyl & $\mathrm{H}$ & $\mathrm{OH}$ & $1987^{1 \mathrm{a}}$ & $1999^{4}$ \\
\hline Isonaamine C (1e) & 4-Methoxybenzyl & $\mathrm{H}$ & $\mathrm{MeO}$ & $1992^{9}$ & $2003^{8}$ \\
\hline Isonaamine D (1g) & 4-Hydroxybenzyl & $\mathrm{H}$ & $\mathrm{MeO}$ & $1998^{3 d}$ & - \\
\hline Isonaamine $\mathrm{E}$ (1f) & 4-Methoxybenzyl & $\mathrm{MeO}$ & $\mathrm{MeO}$ & $2002^{10}$ & - \\
\hline
\end{tabular}

SYNTHESIS 2008, No. 13, pp 2083-2088

Advanced online publication: 11.06.2008

DOI: 10.1055/s-2008-1078444; Art ID: Z07408SS

(c) Georg Thieme Verlag Stuttgart · New York 


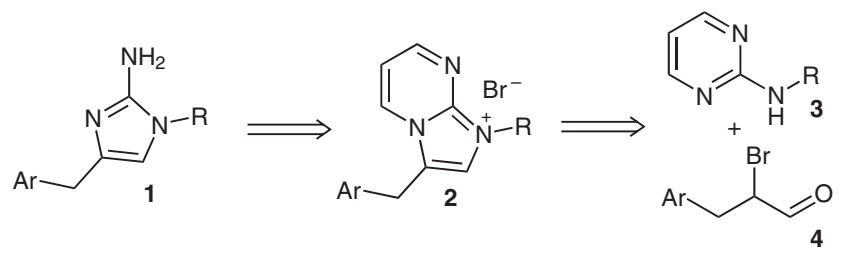

Scheme 1 Retrosynthetic analysis for the synthesis of 1,4-substituted 2-aminoimidazoles

The crucial step in the synthesis of 2-aminoimidazoles from 2-aminopyrimidines is the formation of imidazo[1,2-a]pyrimidin-1-ium salts 2 (Scheme 1). ${ }^{11}$ As a proof of this concept, the cyclization step was initially optimized using 2-methylaminopyrimidine ${ }^{12}$ (3a) and 1.35 equivalents of 2-bromo-3-phenylpropanal (4a) as starting materials (Table 2). At room temperature (or after reflux in $\mathrm{MeCN}$ ) we observed the formation of stable hydrate $\mathbf{5 a}$ instead of the expected aromatic salt $\mathbf{2 a}$. We carefully investigated the microwave-assisted dehydration of the intermediate salt $\mathbf{5 a}$ to the dehydrated salt $\mathbf{2 a}$. A sealed vial containing a solution of the starting compounds $\mathbf{3 a}$ and $\mathbf{4 a}$ in acetonitrile was irradiated at $120-130{ }^{\circ} \mathrm{C}$ for $20-30$ minutes (Table 2, entries 1-3). However, only trace amounts of the desired 1-methyl-3-benzylimidazo[1,2a]pyrimidin-1-ium salt (2a) were observed next to the hydroxy salt 5a. Upon further increasing the temperature to $140{ }^{\circ} \mathrm{C}$, a nearly equimolar mixture of salts $\mathbf{5 a}$ and $\mathbf{2 a}$ was obtained (Table 2, entry 4 ). Increasing the ceiling temperature to $160{ }^{\circ} \mathrm{C}$ and the maximum power to $200 \mathrm{~W}$ for 25 minutes drove the reaction completely to the formation of the desired imidazo[1,2-a]pyrimidin-1-ium salt $\mathbf{2 a}$ as the sole reaction product (Table 2, entry 6).

Having optimized the microwave-assisted protocol for the synthesis of 1,4-substituted 2-aminoimidazoles, we developed a short route for the related marine sponge alkaloids from readily available starting materials. 2-Benzylaminopyrimidine $^{13}$ (3b) and 2-(4-methoxybenzyl)aminopyrimidine (3c) were prepared from the corresponding amines and 2-chloropyrimidine (6) by microwave irradiation (Scheme 2). Subsequent demethylation of the methoxy group of compound 3c, followed by silyl protection with tert-butyldimethylsilyl chloride, provided pyrimidine 8 .

For the synthesis of $\alpha$-bromo aldehydes, the substituted 3phenylpropanols $\mathbf{9 a - d}$, which can be easily accessed from the corresponding cinnamic acids, ${ }^{14}$ were oxidized to the aldehydes 10a-d. ${ }^{15}$ Mild bromination ${ }^{16}$ of $\mathbf{1 0 a}-\mathbf{d}$ using 0.5 equivalent of 5,5-dibromobarbituric acid (DBBA) ${ }^{17}$ at room temperature resulted in the formation of the required
Table 2 Investigation of the Condensation under Conventional Heating and Microwave Irradiation Conditions $\mathrm{s}^{\mathrm{a}}$

\begin{tabular}{|c|c|c|c|c|}
\hline Entry & Temp $\left({ }^{\circ} \mathrm{C}\right)$ & Time $(\min )$ & Power $(\mathrm{W})^{\mathrm{b}}$ & Ratio $\mathbf{5 a}: \mathbf{2 a}^{\mathrm{c}}$ \\
\hline 1 & 120 & 20 & 120 & 100:0 \\
\hline 2 & 130 & 20 & 150 & $95: 5$ \\
\hline 3 & 130 & 30 & 150 & $67: 33$ \\
\hline 4 & 140 & 30 & 150 & $51: 49$ \\
\hline 5 & 150 & 30 & 150 & $8: 92$ \\
\hline 6 & 160 & 25 & 200 & $0: 100$ \\
\hline
\end{tabular}

a All reactions were carried out on a $1 \mathrm{mmol}$ scale of 2-methylaminopyrimidine (3a) with 2-bromo-3-phenylpropan-1-al (4a) (1.35 equiv) in $\mathrm{MeCN}(5 \mathrm{~mL})$.

${ }^{\mathrm{b}}$ Ceiling power of MW irradiation.

${ }^{\mathrm{c}}$ Determined by ${ }^{1} \mathrm{H}$ NMR spectroscopy.

$\alpha$-bromo aldehydes $\mathbf{4 a - d}$ (Scheme 2 ). These were irradiated together with 2-alkylaminopyrimidines $\mathbf{3 a}-\mathbf{c}$ and $\mathbf{8}$ in acetonitrile at $80{ }^{\circ} \mathrm{C}$ for 10 minutes, and subsequently at $160{ }^{\circ} \mathrm{C}$ for 25 minutes, leading to the desired intermediates $\mathbf{2 a}-\mathbf{g}$. The final step - cleavage of the pyrimidine fragment - was achieved by the addition of hydrazine hydrate (7 equiv) to the cooled reaction mixture, and irradiation was continued at $100{ }^{\circ} \mathrm{C}$ for another 10 minutes. The obtained 1,4-substituted 2-aminoimidazoles 1a-g were isolated in good yields as shown in Table 3 (entries 1-7). Remarkably, we observed almost complete loss of the TBDMS group under the cleavage conditions (Table 3, entry 7) and 2-aminoimidazole $\mathbf{1 g}$ was isolated in $58 \%$ yield together with $5 \%$ of the protected counterpart $\mathbf{1 h}$. The 2-aminoimidazoles 1d and 1e were demethylated with $\mathrm{BBr}_{3}$ to give preclathridine $\mathrm{A}(\mathbf{1 i})$ and isonaamine $\mathrm{A}$ (1j) in good yields (Table 3, entries 8 and 9).

Finally, the structure of synthetic isonaamine C (1e) was unambiguously confirmed by single crystal X-ray crystallography (Figure 1). ${ }^{18}$ Interestingly, two hydrogen bonds (between amino groups and endocyclic nitrogen atoms) link two aminoimidazole molecules in the crystal into centrosymmetric dimers (with NH...N bond length 2.07 $\AA$ ), similar to the effect we observed earlier ${ }^{19}$ for 2 -amino1-methyl-5-(4-chlorophenyl)imidazole.

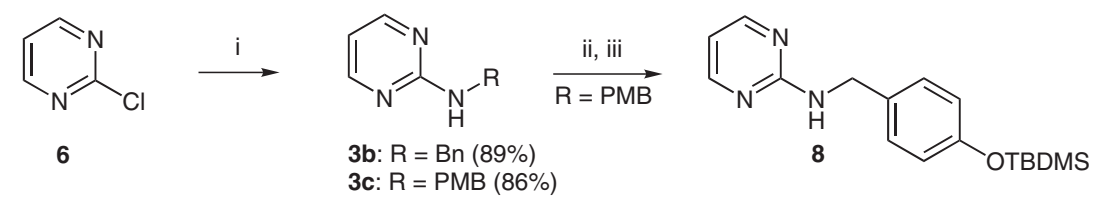

Scheme 2 Reagents and conditions: (i) amine (1.3 equiv), $\mathrm{Et}_{3} \mathrm{~N}$ (1.5 equiv), $\mathrm{EtOH}, \mathrm{MW} 80 \mathrm{~W}, 120^{\circ} \mathrm{C}, 5$ min; (ii) $\mathrm{BBr}_{3}(5 \mathrm{equiv}), \mathrm{CH}_{2} \mathrm{Cl}_{2}$, $0{ }^{\circ} \mathrm{C}$ to r.t., $\left.12 \mathrm{~h} \mathrm{(} \rightarrow \mathbf{7}, 64 \%\right)$; (iii) TBDMSCl (1.25 equiv), imidazole (1.4 equiv), DMF, r.t., overnight (92\%). 

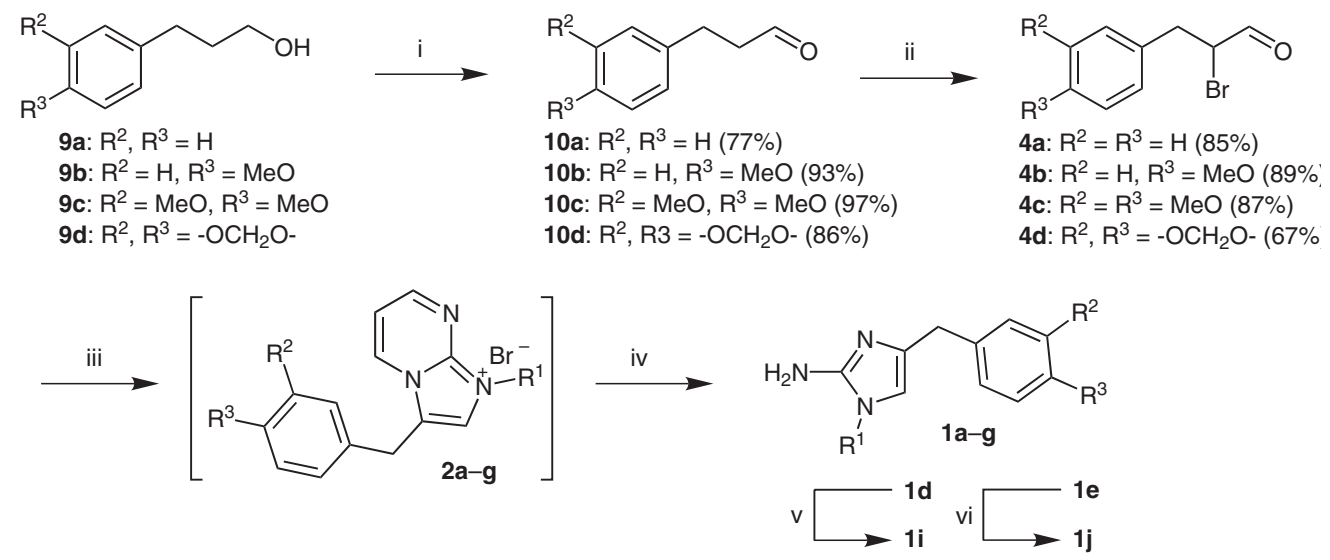

Scheme 3 Reagents and conditions: (i) $\mathrm{PCC}$ (1.4 equiv), $\mathrm{CH}_{2} \mathrm{Cl}_{2}, 0{ }^{\circ} \mathrm{C}, 3-4 \mathrm{~h}$; (ii) $\mathrm{DBBA}$ (0.5 equiv), $\mathrm{Et}_{2} \mathrm{O}, \mathrm{HCl}$ (cat.), r.t., $15-20 \mathrm{hr}$; (iii) 2alkylaminopyrimidines 3a-c and 8, DMAP (cat.), MeCN, MW $200 \mathrm{~W}, 80^{\circ} \mathrm{C}, 10 \mathrm{~min}$, then $160{ }^{\circ} \mathrm{C}, 25 \mathrm{~min}$; (iv) hydrazine hydrate (7 equiv), MW $100 \mathrm{~W}, 100{ }^{\circ} \mathrm{C}, 10 \mathrm{~min}$; (v) $\mathrm{BBr}_{3}$ (5 equiv), $\mathrm{CH}_{2} \mathrm{Cl}_{2}, 0{ }^{\circ} \mathrm{C}$ to r.t., $0.5 \mathrm{~h}$; (vi) $\mathrm{BBr}_{3}$ (10 equiv), $\mathrm{CH}_{2} \mathrm{Cl}_{2}, 0{ }^{\circ} \mathrm{C}$ to r.t., 1 h.

Table 3 2-Aminoimidazoles 1a-j Prepared $^{\mathrm{a}}$

\begin{tabular}{llllll}
\hline Entry & \multicolumn{2}{l}{ Product $\mathrm{R}^{1}$} & $\mathrm{R}^{2}$ & $\mathrm{R}^{3}$ & $\mathrm{Y}$ (ield $(\%)^{\mathrm{b}}$ \\
\hline 1 & $\mathbf{1 a}$ & $\mathrm{Me}$ & $\mathrm{H}$ & $\mathrm{Bn}$ & 88 \\
2 & $\mathbf{1 b}$ & $\mathrm{Bn}$ & $\mathrm{H}$ & $\mathrm{Bn}$ & 74 \\
3 & $\mathbf{1 c}$ & $\mathrm{Me}$ & $-\mathrm{OCH}_{2} \mathrm{O}-$ & 67 \\
4 & $\mathbf{1 d}$ & $\mathrm{Me}$ & $\mathrm{H}$ & $\mathrm{MeO}$ & 87 \\
5 & $\mathbf{1 e}$ & $4-$ Methoxybenzyl & $\mathrm{H}$ & $\mathrm{MeO}$ & 89 \\
6 & $\mathbf{1 f}$ & $4-$ Methoxybenzyl & $\mathrm{MeO}$ & $\mathrm{MeO}$ & 85 \\
7 & $\mathbf{1 g}$ & $4-$ Hydroxybenzyl & $\mathrm{H}$ & $\mathrm{MeO}$ & 58 \\
& + & + & & & + \\
& $\mathbf{1 h}$ & $4-\mathrm{TBDMSObenzyl}$ & & & 5 \\
8 & $\mathbf{1 i}$ & Me & $\mathrm{H}$ & $\mathrm{OH}$ & 55 \\
9 & $\mathbf{1 j}$ & $4-$ Hydroxybenzyl & $\mathrm{H}$ & $\mathrm{OH}$ & 71 \\
\hline
\end{tabular}

${ }^{a}$ Yields of 2-aminoimidazoles 1a-h given for the one-pot procedure, starting from $\alpha$-bromo aldehydes $\mathbf{4 a - d}$.

${ }^{\mathrm{b}}$ Isolated yield.

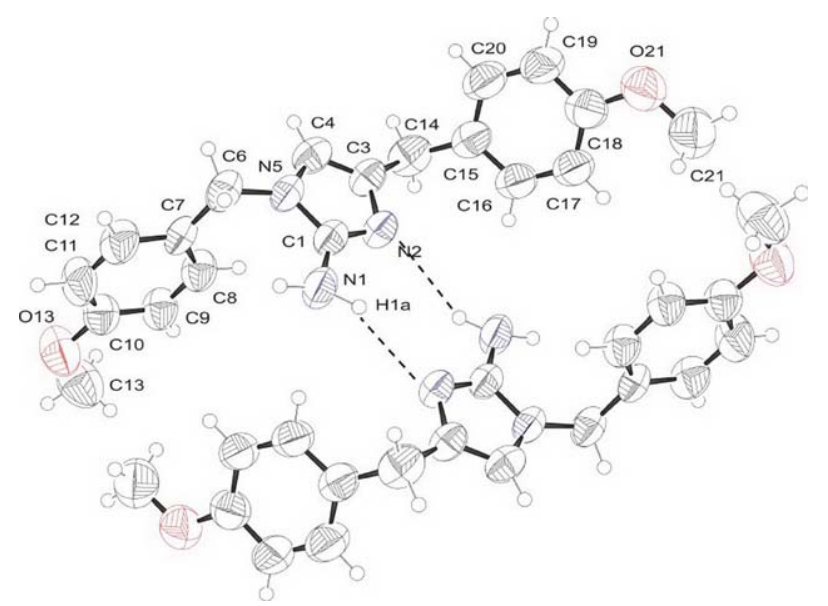

Figure 1 Crystal structure of isonaamine $\mathrm{C}$
In conclusion, we have applied a short and efficient microwave-assisted protocol for the preparation of the 1,4dialkyl-2-aminoimidazole-based marine sponge alkaloids, using readily available substituted 2-aminopyrimidines as the masked guanidine function ${ }^{20}$ in the reaction with $\alpha$-bromo aldehydes. In addition to its simplicity, this method provides high yields of products in short reaction times. The microwave-assisted procedure would be of great use in the synthesis of a range of 2-aminoimidazolebased natural products.

Melting points were determined using a Reichert-Jung Thermovar apparatus or an Electrothermal 9200 digital melting point apparatus and are uncorrected. ${ }^{1} \mathrm{H}$ NMR spectra were recorded on a Bruker Avance $300(300 / 75.5 \mathrm{MHz})$ and $400(400 / 100.5 \mathrm{MHz})$ instruments using $\mathrm{CDCl}_{3}$ and DMSO- $d_{6}$ as solvents. The ${ }^{1} \mathrm{H}$ and ${ }^{13} \mathrm{C}$ chemical shifts are reported in parts per million relative to tetramethylsilane using the residual solvent signal as an internal reference. Mass spectra were recorded by using a Kratos MS 50 TC, Kratos Mach III system, and LCQ Advantage (Thermo Electron Corp.). The ion source temperature was $150-250{ }^{\circ} \mathrm{C}$, as required. High-resolution EI-mass spectra were performed with a resolution of 10000 . The low-resolution spectra were obtained with a HP5989A MS instrument. For TLC, analytical TLC plates [Alugram SIL G/UV ${ }_{254}$ and 70-230 mesh silica gel (E. M. Merck)] were used.

\section{Microwave Experiments}

A multimode Milestone MicroSYNTH microwave reactor (Laboratory Microwave Systems) was used in the standard configuration as delivered, including proprietary software. Reaction temperatures were monitored by an IR sensor on the outside wall of the reaction vial and a fiber optic sensor inside the reaction vial. All experiments were carried out in sealed microwave process vials $(15,50 \mathrm{~mL})$. After completion of the reaction, the vial was cooled to $25^{\circ} \mathrm{C}$ via air jet cooling before opening.

\section{(4-Methoxybenzyl)pyrimidin-2-ylamine (3c)}

In a $50 \mathrm{~mL}$ microwave vial, 2-chloropyrimidine $(3.43 \mathrm{~g}, 30 \mathrm{mmol})$, 4-methoxybenzylamine (5.35 g, 39 mmol, 1.3 equiv), and $\mathrm{Et}_{3} \mathrm{~N}(6.2$ $\mathrm{mL}, 45 \mathrm{mmol}, 1.5$ equiv) were successively dissolved in EtOH (20 $\mathrm{mL}$ ). The reaction tube was sealed, and irradiated in the cavity of a microwave reactor at a ceiling temperature of $120^{\circ} \mathrm{C}$ at $80 \mathrm{~W}$ maximum power for $5 \mathrm{~min}$. After the mixture was cooled with an air flow for $15 \mathrm{~min}$, it was diluted with $\mathrm{H}_{2} \mathrm{O}(100 \mathrm{~mL})$, extracted with $\mathrm{CH}_{2} \mathrm{Cl}_{2}(2 \times 150 \mathrm{~mL})$, and the combined organic extracts were dried 
$\left(\mathrm{Na}_{2} \mathrm{SO}_{4}\right)$. The solvent was removed under reduced pressure and the residue was subjected to silica gel flash chromatography $(0-5 \%$ $\mathrm{MeOH}-\mathrm{CH}_{2} \mathrm{Cl}_{2}$ ) to afford $5.55 \mathrm{~g}(86 \%)$ of $\mathbf{3 c}$; colorless solid; $\mathrm{mp}$ $101-102{ }^{\circ} \mathrm{C}$.

${ }^{1} \mathrm{H}$ NMR $\left(300 \mathrm{MHz}, \mathrm{CDCl}_{3}\right): \delta=8.26(\mathrm{~d}, J=4.8 \mathrm{~Hz}, 2 \mathrm{H}), 7.26(\mathrm{~d}$, $J=8.6 \mathrm{~Hz}, 2 \mathrm{H}), 6.86(\mathrm{~d}, J=8.5 \mathrm{~Hz}, 2 \mathrm{H}), 6.54(\mathrm{t}, J=4.8 \mathrm{~Hz}, 1 \mathrm{H})$, $5.50(\mathrm{br}, 1 \mathrm{H}), 4.56(\mathrm{~d}, J=5.7 \mathrm{~Hz}, 2 \mathrm{H}), 3.81(\mathrm{~s}, 3 \mathrm{H})$.

${ }^{13} \mathrm{C} \mathrm{NMR}\left(75.5 \mathrm{MHz}, \mathrm{CDCl}_{3}\right): \delta=162.7,159.3,158.4(2 \times), 131.6$, $129.3(2 \times), 114.4(2 \times), 110.9,55.7,45.4$.

HRMS-EI: $\mathrm{m} / z$ calcd for $\mathrm{C}_{16} \mathrm{H}_{21} \mathrm{~N}_{3}[\mathrm{M}]^{+}$: 215.1059; found: 215.1064.

\section{4-(Pyrimidin-2-ylaminomethyl)phenol (7)}

To a solution of $3 \mathbf{c}(3.23 \mathrm{~g}, 15 \mathrm{mmol})$ in $\mathrm{CH}_{2} \mathrm{Cl}_{2}(50 \mathrm{~mL})$ at $0{ }^{\circ} \mathrm{C}$ was added dropwise $\mathrm{BBr}_{3}(7.2 \mathrm{~mL}, 75 \mathrm{mmol}, 5$ equiv) and the mixture was stirred at r.t. for 1 day. The mixture was cooled in an ice bath, and then $6 \mathrm{~N}$ ammonia in $\mathrm{MeOH}(\sim 60 \mathrm{~mL})$ was added carefully. The mixture was diluted with $\mathrm{H}_{2} \mathrm{O}(200 \mathrm{~mL})$, extracted with EtOAc $(2 \times 150 \mathrm{~mL})$, and the combined organic extracts were dried $\left(\mathrm{Na}_{2} \mathrm{SO}_{4}\right)$. The solvent was removed under reduced pressure and the residue was subjected to column chromatography on silica gel (from $10 \% \mathrm{MeOH}-\mathrm{CH}_{2} \mathrm{Cl}_{2}$ ) to afford $1.93 \mathrm{~g} \mathrm{(64 \% )} \mathrm{of} \mathrm{7;} \mathrm{white} \mathrm{solid;}$ mp $183-185{ }^{\circ} \mathrm{C}$.

${ }^{1} \mathrm{H}$ NMR $\left(300 \mathrm{MHz}, \mathrm{DMSO}-d_{6}\right): \delta=9.22(\mathrm{~s}, 1 \mathrm{H}), 8.24(\mathrm{~d}, J=4.7$ $\mathrm{Hz}, 2 \mathrm{H}), 7.54(\mathrm{t}, J=6.0 \mathrm{~Hz}, 1 \mathrm{H}), 7.10(\mathrm{~d}, J=8.2 \mathrm{~Hz}, 2 \mathrm{H}), 6.67(\mathrm{~d}$, $J=8.2 \mathrm{~Hz}, 2 \mathrm{H}), 6.54(\mathrm{t}, J=4.8 \mathrm{~Hz}, 1 \mathrm{H}), 4.36(\mathrm{~d}, J=6.3 \mathrm{~Hz}, 2 \mathrm{H})$.

${ }^{13} \mathrm{C}$ NMR $\left(75.5 \mathrm{MHz}\right.$, DMSO- $\left.d_{6}\right): \delta=163.1,158.9,158.8,156.9$, 131.4, 129.3, 115.8, 110.9, 44.3.

HRMS-EI: $\mathrm{m} / \mathrm{z}$ calcd for $\mathrm{C}_{16} \mathrm{H}_{21} \mathrm{~N}_{3}[\mathrm{M}]^{+}:$201.0902; found: 201.0892 .

[4-(tert-Butyldimethylsilyloxy)benzyl]pyrimidin-2-yl-amine (8) To a solution of $7(1.4 \mathrm{~g}, 7 \mathrm{mmol})$ in DMF $(25 \mathrm{~mL})$ were added TBDMSCl (1.32 g, $8.75 \mathrm{mmol}, 1.25$ equiv) and imidazole $(0.67 \mathrm{~g}$, $9.8 \mathrm{mmol}, 1.4$ equiv), and the mixture was stirred overnight at $\mathrm{r} . \mathrm{t}$. After partition of the mixture between $\mathrm{Et}_{2} \mathrm{O}(200 \mathrm{~mL})$ and aq sat. $\mathrm{NaHCO}_{3}(100 \mathrm{~mL})$, the organic layer was washed with $\mathrm{H}_{2} \mathrm{O}(50$ $\mathrm{mL})$ and brine $(50 \mathrm{~mL})$, dried $\left(\mathrm{MgSO}_{4}\right)$, and concentrated under reduced pressure. The crude product was purified by flash chromatography on neutral alumina $\left(\mathrm{CH}_{2} \mathrm{Cl}_{2}-\mathrm{Et}_{2} \mathrm{O}, 4: 1\right)$ to afford $2.03 \mathrm{~g}(92 \%)$ of 8; colorless solid; mp 132-134 ${ }^{\circ} \mathrm{C}$.

${ }^{1} \mathrm{H}$ NMR $\left(300 \mathrm{MHz}, \mathrm{CDCl}_{3}\right): \delta=8.28(\mathrm{~d}, J=4.2 \mathrm{~Hz}, 2 \mathrm{H}), 7.21(\mathrm{~d}$, $J=8.3 \mathrm{~Hz}, 2 \mathrm{H}), 6.80(\mathrm{~d}, J=8.6 \mathrm{~Hz}, 2 \mathrm{H}), 6.55(\mathrm{t}, J=4.7 \mathrm{~Hz}, 1 \mathrm{H})$, 5.49 (br, $1 \mathrm{H}), 4.56(\mathrm{~d}, J=5.5 \mathrm{~Hz}, 2 \mathrm{H}), 0.99(\mathrm{~s}, 9 \mathrm{H}), 0.20(\mathrm{~s}, 6 \mathrm{H})$.

${ }^{13} \mathrm{C} \mathrm{NMR}\left(75.5 \mathrm{MHz}, \mathrm{CDCl}_{3}\right): \delta=162.7,158.4(2 \times), 155.3,132.1$, $129.3(2 \times), 120.6(2 \times), 111.0,45.5,26.1(3 \times), 18.6,-4.0(2 \times)$.

HRMS-EI: $\mathrm{m} / \mathrm{z}$ calcd for $\mathrm{C}_{16} \mathrm{H}_{21} \mathrm{~N}_{3}[\mathrm{M}]^{+}:$315.1767; found: 315.1768 .

\section{Compounds 2a and 5a}

In a $15 \mathrm{~mL}$ microwave vial, 2-methylaminopyrimidine (3a; $109 \mathrm{mg}$, $1 \mathrm{mmol}$ ) and 2-bromo-3-phenylpropanal (4a; $288 \mathrm{mg}, 1.35 \mathrm{mmol}$, 1.35 equiv) were successively dissolved in $\mathrm{MeCN}$. The reaction tube was flushed with argon, sealed, and irradiated in the cavity of a microwave reactor at $80 \mathrm{~W}$ and at a ceiling temperature specified in Table 2. After the mixture was cooled with an air flow for $10 \mathrm{~min}$, it was diluted with $\mathrm{H}_{2} \mathrm{O}(25 \mathrm{~mL})$, and the precipitate was washed with a 1:1 mixture $\mathrm{Et}_{2} \mathrm{O}$-acetone $(2 \times 10 \mathrm{~mL})$ and dried in vacuum.

3-Benzyl-2-hydroxy-1-methyl-1H,2H,3H-imidazo[1,2-a]pyrimidin-4-ium Bromide (5a) (Table 2, Entry 1)

White solid; mp $197-199^{\circ} \mathrm{C}$.
${ }^{1} \mathrm{H} \mathrm{NMR}\left(300 \mathrm{MHz}, \mathrm{CDCl}_{3}\right): \delta=8.97(\mathrm{~m}, 1 \mathrm{H}), 8.81(\mathrm{~m}, 1 \mathrm{H}), 7.42$ $7.23(\mathrm{~m}, 7 \mathrm{H}), 5.32(\mathrm{dd}, J=8.3,1.8 \mathrm{~Hz}, 1 \mathrm{H}), 4.93(\mathrm{~m}, 1 \mathrm{H}), 3.32(\mathrm{~s}$, $2 \mathrm{H}), 3.08$ (s, $3 \mathrm{H})$.

${ }^{13} \mathrm{C}$ NMR $\left(75.5 \mathrm{MHz}, \mathrm{CDCl}_{3}\right): \delta=168.9,154.8,148.5,135.0,130.6$ $(2 \times), 129.6(2 \times), 128.3,111.6,86.1,68.7,37.8,28.9$.

\section{3-Benzyl-1-methylimidazo[1,2-a]pyrimidin-1-ium Bromide (2a) (Table 2, Entry 6) \\ Colorless solid; $\mathrm{mp} 155-156^{\circ} \mathrm{C}$.}

${ }^{1} \mathrm{H}$ NMR $\left(300 \mathrm{MHz}, \mathrm{CDCl}_{3}\right): \delta=9.28(\mathrm{~m}, 1 \mathrm{H}), 9.12(\mathrm{~m}, 1 \mathrm{H}), 8.16$ $(\mathrm{s}, 1 \mathrm{H}), 7.74(\mathrm{dd}, J=6.3,4.8 \mathrm{~Hz}, 1 \mathrm{H}), 7.38-7.30(\mathrm{~m}, 5 \mathrm{H}), 4.46$ (s, $2 \mathrm{H}), 4.01$ (s, $3 \mathrm{H})$.

${ }^{13} \mathrm{C}$ NMR (75.5 MHz, $\left.\mathrm{CDCl}_{3}\right): \delta=157.9,143.0,137.2,136.0,129.7$ $(4 \times), 128.1,125.1,124.9,114.4,33.6,29.1$.

DEPT NMR (75.5 MHz, $\left.\mathrm{CDCl}_{3}\right): \delta=157.9,137.2,129.7(4 \times)$, 128.1, 125.1, 114.4, 80.0, 33.6, 29.1.

\section{3-Aryl-2-bromopropanals 4a-d; 2-Bromo-3-phenylpropanal (4a); Typical Procedure}

A solution of $10 \mathrm{a}(1.34 \mathrm{~g}, 10 \mathrm{mmol})$ in $\mathrm{Et}_{2} \mathrm{O}(10 \mathrm{~mL})$ was added dropwise to a solution of DBBA (1.45 g, 5 mmol, 5 equiv) in $\mathrm{Et}_{2} \mathrm{O}$ $(40 \mathrm{~mL})$. Then, a $4 \mathrm{~N}$ solution of $\mathrm{HCl}$ in 1,4-dioxane $(0.25 \mathrm{~mL}, 1$ $\mathrm{mmol}$ ) was added dropwise and the mixture was stirred for $15 \mathrm{~h}$ at r.t. After partition of the mixture between $\mathrm{Et}_{2} \mathrm{O}(150 \mathrm{~mL})$ and aq sat. $\mathrm{NaHCO}_{3}(100 \mathrm{~mL})$, the organic layer was washed with $\mathrm{H}_{2} \mathrm{O}(50$ $\mathrm{mL})$ and brine $(50 \mathrm{~mL})$, dried $\left(\mathrm{MgSO}_{4}\right)$, and concentrated under reduced pressure to give a light yellow oil. The crude product was purified by flash chromatography on neutral alumina $\left(\mathrm{CH}_{2} \mathrm{Cl}_{2}-\mathrm{Et}_{2} \mathrm{O}\right.$, $2: 1)$ to afford $1.81 \mathrm{~g}(85 \%)$ of $\mathbf{4 a}$; light oil.

${ }^{1} \mathrm{H}$ NMR (300 MHz, $\left.\mathrm{CDCl}_{3}\right): \delta=9.51(\mathrm{~s}, 1 \mathrm{H}), 7.38-7.24(\mathrm{~m}, 5 \mathrm{H})$, $4.47(\mathrm{~m}, 1 \mathrm{H}), 3.55-3.48(\mathrm{~m}, 1 \mathrm{H}), 3.24-3.16(\mathrm{~m}, 1 \mathrm{H})$.

${ }^{13} \mathrm{C} \mathrm{NMR}\left(75.5 \mathrm{MHz}, \mathrm{CDCl}_{3}\right): \delta=192.3,136.7,129.7(2 \times), 129.2$ $(2 \times), 127.8,55.1,38.4$.

HRMS-EI: $\mathrm{m} / \mathrm{z}$ calcd for $\mathrm{C}_{9} \mathrm{H}_{9} \mathrm{BrO}[\mathrm{M}]^{+}:$211.9837; found: 211.9850 .

\section{2-Bromo-3-(4-methoxyphenyl)propanal (4b)}

Yield: $89 \%$; light oil.

${ }^{1} \mathrm{H}$ NMR (300 MHz, $\left.\mathrm{CDCl}_{3}\right): \delta=9.49(\mathrm{~s}, 1 \mathrm{H}), 7.19(\mathrm{~d}, J=8.4 \mathrm{~Hz}$, $2 \mathrm{H}), 6.86(\mathrm{~d}, J=8.4 \mathrm{~Hz}, 2 \mathrm{H}), 4.86(\mathrm{~m}, 1 \mathrm{H}), 3.73(\mathrm{~s}, 1 \mathrm{H}), 3.39(\mathrm{~m}$, $1 \mathrm{H}), 3.14(\mathrm{~m}, 1 \mathrm{H})$.

${ }^{13} \mathrm{C} \mathrm{NMR}\left(75.5 \mathrm{MHz}, \mathrm{CDCl}_{3}\right): \delta=192.5,159.3,130.8(2 \times), 128.6$, $114.6(2 \times), 55.7,55.4,37.7$.

HRMS-EI: $m / z$ calcd for $\mathrm{C}_{10} \mathrm{H}_{11} \mathrm{BrO}_{2}[\mathrm{M}]^{+}:$241.9942; found: 241.9939.

\section{2-Bromo-3-(3,4-dimethoxyphenyl)propanal (4c)}

Yield: 87\%; light oil.

${ }^{1} \mathrm{H}$ NMR $\left(300 \mathrm{MHz}, \mathrm{CDCl}_{3}\right): \delta=9.48(\mathrm{~s}, 1 \mathrm{H}), 6.92-6.74(\mathrm{~m}, 3 \mathrm{H})$, $4.43(\mathrm{~m}, 1 \mathrm{H}), 3.87$ (s, $3 \mathrm{H}), 3.86$ (s, $3 \mathrm{H}), 3.46-3.39(\mathrm{~m}, 1 \mathrm{H}), 3.17-$ $3.10(\mathrm{~m}, 1 \mathrm{H})$.

${ }^{13} \mathrm{C}$ NMR $\left(75.5 \mathrm{MHz}, \mathrm{CDCl}_{3}\right): \delta=192.5,149.5,148.8,129.1$, 121.9, 112.8, 111.7, 68.4, 56.3, 55.2, 38.1.

HRMS-EI: $\mathrm{m} / \mathrm{z}$ calcd for $\mathrm{C}_{11} \mathrm{H}_{13} \mathrm{BrO}_{3}[\mathrm{M}]^{+}:$272.0048; found: 272.0043.

\section{3-(1,3-Benzodioxol-5-yl)-2-bromopropanal (4d)}

Yield: 67\%; light oil.

${ }^{1} \mathrm{H}$ NMR $\left(300 \mathrm{MHz}, \mathrm{CDCl}_{3}\right): \delta=9.48(\mathrm{~s}, 1 \mathrm{H}), 6.75-6.68(\mathrm{~m}, 3 \mathrm{H})$, $6.01(\mathrm{~s}, 2 \mathrm{H}), 4.42(\mathrm{~m}, 1 \mathrm{H}), 3.64-3.50(\mathrm{~m}, 1 \mathrm{H}), 3.18-3.09(\mathrm{~m}, 1 \mathrm{H})$.

${ }^{13} \mathrm{C}$ NMR $\left(75.5 \mathrm{MHz}, \mathrm{CDCl}_{3}\right): \delta=192.0,149.7,148.5,129.7$, 121.6, 109.3, 108.7, 101.4, 58.7, 38.6. 
HRMS-EI: $m / z$ calcd for $\mathrm{C}_{10} \mathrm{H}_{9} \mathrm{BrO}_{3}[\mathrm{M}]^{+}:$255.9735; found: 255.9738 .

Microwave-Assisted Synthesis of 1a-g; 4-(4-Benzylbenzyl)-1methyl-1H-imidazol-2-ylamine (1a); Typical Procedure A $10 \mathrm{~mL}$ microwave vial was successively charged with $\mathrm{MeCN}(10$ $\mathrm{mL}$ ), 2-methylaminopyrimidine (3a; $435 \mathrm{mg}, 4 \mathrm{mmol}$ ), 2-bromo-3phenylpropanal (4a; $1.15 \mathrm{~g}, 5.4 \mathrm{mmol}, 1.35$ equiv), and DMAP (5 $\mathrm{mg}, 0.04 \mathrm{mmol}, 1 \mathrm{~mol} \%)$. The reaction tube was sealed, and irradiated in a microwave reactor first at a ceiling temperature of $80^{\circ} \mathrm{C}$ at $200 \mathrm{~W}$ maximum power for $10 \mathrm{~min}$, and then at $160{ }^{\circ} \mathrm{C}$ at $200 \mathrm{~W}$ maximum power for $25 \mathrm{~min}$. After the mixture was cooled with an air flow for $15 \mathrm{~min}$, hydrazine hydrate $(1.4 \mathrm{~mL}$ of a $64 \%$ solution, $28 \mathrm{mmol} 7$ equiv) was added, and the mixture was irradiated for another 10 min at a ceiling temperature of $100{ }^{\circ} \mathrm{C}$ at $100 \mathrm{~W}$ maximum power. The mixture was diluted with $\mathrm{CH}_{2} \mathrm{Cl}_{2}(150 \mathrm{~mL})$, the $\mathrm{CH}_{2} \mathrm{Cl}_{2}$ layer was washed with aq sat. $\mathrm{NH}_{4} \mathrm{Cl}(100 \mathrm{~mL})$, brine $(100 \mathrm{~mL})$, and $\mathrm{H}_{2} \mathrm{O}(2 \times 100 \mathrm{~mL})$ and dried $\left(\mathrm{Na}_{2} \mathrm{SO}_{4}\right)$. After filtration and concentration, the resulting residue was purified by column chromatography (silica gel; $\mathrm{CH}_{2} \mathrm{Cl}_{2}-\mathrm{MeOH}, 9: 1$ with $3 \% \mathrm{Et}_{3} \mathrm{~N}$ ) to afford $659 \mathrm{mg}$ (88\%) of 1a; light yellow solid; $\mathrm{mp} 66-68{ }^{\circ} \mathrm{C}$.

${ }^{1} \mathrm{H}$ NMR $\left(300 \mathrm{MHz}, \mathrm{CDCl}_{3}\right): \delta=7.28(\mathrm{~m}, 4 \mathrm{H}), 7.18(\mathrm{~m}, 1 \mathrm{H}), 6.09$ (s, $1 \mathrm{H}), 4.16$ (br, $2 \mathrm{H}), 3.76$ (s, $2 \mathrm{H}), 3.30$ (s, $3 \mathrm{H})$.

${ }^{13} \mathrm{C}$ NMR $\left(75 \mathrm{MHz}, \mathrm{CDCl}_{3}\right): \delta=148.1,140.8,137.4,129.3(2 \times)$, $128.7(2 \times), 126.3,113.3,35.4,31.6$.

HRMS-EI: $\mathrm{m} / \mathrm{z}$ calcd for $\mathrm{C}_{11} \mathrm{H}_{13} \mathrm{~N}_{3}[\mathrm{M}]^{+}$: 187.1109; found: 187.1108.

\section{1-Benzyl-4-(4-benzylbenzyl)-1 $H$-imidazol-2-ylamine (1b)}

Yield: $74 \%$; light yellow solid; $\mathrm{mp} 124-125^{\circ} \mathrm{C}$.

${ }^{1} \mathrm{H}$ NMR $\left(300 \mathrm{MHz}, \mathrm{CDCl}_{3}\right): \delta=7.30(\mathrm{~m}, 7 \mathrm{H}), 7.15(\mathrm{~m}, 3 \mathrm{H}), 6.19$ (s, $1 \mathrm{H}), 4.80(\mathrm{~s}, 2 \mathrm{H}), 4.19(\mathrm{br}, 2 \mathrm{H}), 3.80(\mathrm{~s}, 2 \mathrm{H})$.

${ }^{13} \mathrm{C}$ NMR $\left(75 \mathrm{MHz}, \mathrm{CDCl}_{3}\right): \delta=148.4,140.8,137.5,136.8,129.4$, 128.7, 128.3, 127.2, 126.3, 112.8, 48.8, 35.4.

DEPT NMR $\left(75 \mathrm{MHz}, \mathrm{CDCl}_{3}\right): \delta=129.4(2 \times 2), 129.3(2 \times 2)$, $128.7(2 \times 2), 128.4,127.2(2 \times 2), 126.3,-48.9,-35.4$.

HRMS-EI: $m / z$ calcd for $\mathrm{C}_{17} \mathrm{H}_{17} \mathrm{~N}_{3}[\mathrm{M}]^{+}:$263.1422; found: 263.1425 .

\section{4-(1,3-Benzodioxol-5-ylmethyl)-1-methyl-1H-imidazol-2-yl- amine (1c) \\ Yield: $87 \%$; yellow oil}

${ }^{1} \mathrm{H}$ NMR (400 MHz, DMSO- $\left.d_{6}\right): \delta=6.78(\mathrm{~m}, 2 \mathrm{H}), 6.68(\mathrm{dd}$, $J=8.0,0.9 \mathrm{~Hz}, 1 \mathrm{H}), 6.19(\mathrm{~s}, 1 \mathrm{H}), 5.94(\mathrm{~s}, 2 \mathrm{H}), 5.45(\mathrm{br}, 2 \mathrm{H}), 3.57$ (s, $2 \mathrm{H}), 2.67$ (s, $3 \mathrm{H})$.

${ }^{13} \mathrm{C}$ NMR $\left(75.5 \mathrm{MHz}, \mathrm{DMSO}-d_{6}\right): \delta=151.7,148.0,146.3,133.8$, 121.9, 112.4, 109.6, 108.5, 107.0, 101.2, 33.6, 30.9.

HRMS-EI: $\mathrm{m} / \mathrm{z}$ calcd for $\mathrm{C}_{12} \mathrm{H}_{13} \mathrm{~N}_{3} \mathrm{O}_{2}[\mathrm{M}]^{+}:$231.1008; found: 231.1008.

\section{4-(4-Methoxybenzyl)-1-methyl- $1 H$-imidazol-2-ylamine (1d)}

Yield: 67\%; light brown solid; $\mathrm{mp} 134-136^{\circ} \mathrm{C}$.

${ }^{1} \mathrm{H}$ NMR (400 MHz, DMSO- $d_{6}$ ): $\delta=7.11(\mathrm{~d}, J=8.4 \mathrm{~Hz}, 2 \mathrm{H}), 6.80$ (d, $J=8.4 \mathrm{~Hz}, 2 \mathrm{H}), 6.12$ (s, $1 \mathrm{H}), 5.19$ (br, $2 \mathrm{H}), 3.70$ (s, $3 \mathrm{H}), 3.49$ (s, $2 \mathrm{H}), 3.22$ (s, $3 \mathrm{H})$.

${ }^{13} \mathrm{C}$ NMR $\left(100 \mathrm{MHz}\right.$, DMSO- $\left.d_{6}\right): \delta=157.9,147.7,136.9,132.3$, 129.8, 113.8, 112.7, 55.3, 33.9, 31.3.

HRMS-EI: $\mathrm{m} / \mathrm{z}$ calcd for $\mathrm{C}_{12} \mathrm{H}_{15} \mathrm{~N}_{3} \mathrm{O}[\mathrm{M}]^{+}:$217.1215; found: 263.1218 .

1,4-Bis-(4-methoxybenzyl)-1H-imidazol-2-ylamine (1e) Yield: $89 \%$; colorless solid; mp $139-141^{\circ} \mathrm{C}$.
${ }^{1} \mathrm{H}$ NMR (300 MHz, DMSO- $\left.d_{6}\right): \delta=7.81(\mathrm{br}, 1 \mathrm{H}), 7.13(\mathrm{~m}, 4 \mathrm{H})$, $6.87(\mathrm{~d}, J=8.4 \mathrm{~Hz}, 2 \mathrm{H}), 6.79(\mathrm{~d}, J=8.2 \mathrm{~Hz}, 2 \mathrm{H}), 6.26(\mathrm{~s}, 1 \mathrm{H})$, 6.12 (s, $1 \mathrm{H}), 5.35$ (s, $2 \mathrm{H}), 4.76$ (s, $2 \mathrm{H}), 3.71(\mathrm{~s}, 3 \mathrm{H}), 3.69$ (s, $3 \mathrm{H})$.

${ }^{13} \mathrm{C}$ NMR $\left(75.5 \mathrm{MHz}, \mathrm{DMSO}-d_{6}\right): \delta=159.4,158.2,149.6,137.2$, 133.8, 130.8, $130.4(2 \times), 129.7(2 \times), 114.7(2 \times), 114.3(2 \times)$, 110.9, 105.1, 55.9, 55.8, 47.3, 34.6.

HRMS-EI: $m / z$ calcd for $\mathrm{C}_{20} \mathrm{H}_{23} \mathrm{~N}_{3} \mathrm{O}_{3}[\mathrm{M}]^{+}:$323.1634; found: 323.1631 .

4-(3,4-Dimethoxybenzyl)-1-(4-methoxybenzyl)-1H-imidazol-2ylamine (1f)

Yield: 85\%; brown oil.

${ }^{1} \mathrm{H}$ NMR $\left(300 \mathrm{MHz}, \mathrm{DMSO}-d_{6}\right): \delta=7.19(\mathrm{~d}, J=8.0 \mathrm{~Hz}, 2 \mathrm{H}), 6.80$ (m, $5 \mathrm{H}), 6.17(\mathrm{~s}, 1 \mathrm{H}), 4.58(\mathrm{br}, 2 \mathrm{H}), 4.24(\mathrm{~s}, 2 \mathrm{H}), 3.83(\mathrm{~s}, 3 \mathrm{H})$, 3.80 (s, $3 \mathrm{H}), 3.77$ (s, $3 \mathrm{H}), 3.68$ (s, $2 \mathrm{H}$ ).

${ }^{13} \mathrm{C}$ NMR $\left(75.5 \mathrm{MHz}\right.$, DMSO- $\left.d_{6}\right): \delta=158.9,150.3,148.8,147.5$, $131.8,131.7,130.5,128.5,120.7,114.0,112.1,111.8,111.2,55.9$, $55.8,55.2,47.2,32.6$.

DEPT NMR (75.5 MHz, DMSO- $\left.d_{6}\right): \delta=128.8,121.0,114.3$, $112.5,112.2,11.6,56.3,56.2,55.6,-47.5,-32.96$.

HRMS-EI: $m / z$ calcd for $\mathrm{C}_{20} \mathrm{H}_{23} \mathrm{~N}_{3} \mathrm{O}_{3}$ : [M] $]^{+}$: 353.1739; found: 353.1736 .

4-[2-Amino-4-(4-methoxybenzyl)-1H-imidazol-1-ylmethyl]phenol (1g)

Yield: 58\%; yellow solid; mp $155-157^{\circ} \mathrm{C}$.

${ }^{1} \mathrm{H}$ NMR $\left(300 \mathrm{MHz}, \mathrm{DMSO}-d_{6}\right): \delta=9.33(\mathrm{br}, 1 \mathrm{H}), 7.11(\mathrm{~d}, J=8.6$ $\mathrm{Hz}, 2 \mathrm{H}), 7.02$ (d, $J=8.2 \mathrm{~Hz}, 2 \mathrm{H}), 6.79(\mathrm{~d}, J=8.6 \mathrm{~Hz}, 2 \mathrm{H}), 6.70$ $(\mathrm{d}, J=8.2 \mathrm{~Hz}, 2 \mathrm{H}), 6.10(\mathrm{~s}, 1 \mathrm{H}), 5.23(\mathrm{~s}, 2 \mathrm{H}), 4.70(\mathrm{~s}, 2 \mathrm{H}), 3.70$ (s, $3 \mathrm{H}), 3.49$ (s, $2 \mathrm{H})$.

${ }^{13} \mathrm{C}$ NMR (75.5 MHz, DMSO- $\left.d_{6}\right): \delta=157.3,156.6,148.6,136.2$, 132.9, $129.6(2 \times), 128.9(2 \times), 128.2,115.2(2 \times), 113.4(2 \times)$, $110.0,55.0,46.5,33.7$.

HRMS-EI: $m / z$ calcd for $\mathrm{C}_{18} \mathrm{H}_{19} \mathrm{~N}_{3} \mathrm{O}_{2}[\mathrm{M}]^{+}:$309.1477; found: 309.1482 .

1-[4-(tert-Butyldimethylsilyloxy)benzyl]-4-(4-methoxybenzyl)$1 H$-imidazol-2-ylamine (1h)

Yield: 5\%; white solid; $\mathrm{mp} 115-117^{\circ} \mathrm{C}$.

${ }^{1} \mathrm{H}$ NMR $\left(300 \mathrm{MHz}, \mathrm{DMSO}-d_{6}\right): \delta=7.09(\mathrm{~m}, 4 \mathrm{H}), 6.80(\mathrm{~m}, 4 \mathrm{H})$, 6.12 (s, $1 \mathrm{H}), 5.30$ (br, $2 \mathrm{H}), 4.74$ (s, $2 \mathrm{H}), 3.69$ (s, $3 \mathrm{H}), 3.49$ (s, 2 $\mathrm{H}), 0.93$ (s, $9 \mathrm{H}), 0.16(\mathrm{~s}, 6 \mathrm{H})$.

${ }^{13} \mathrm{C}$ NMR $\left(75.5 \mathrm{MHz}\right.$, DMSO- $\left.d_{6}\right): \delta=158.0,155.8,149.4,137.7$, $130.3,129.9,128.3,120.7,114.1,113.9,112.7,108.1,55.4,48.3$, $34.2,25.8,18.3,-4.3$.

HRMS-EI: $m / z$ calcd for $\mathrm{C}_{24} \mathrm{H}_{23} \mathrm{~N}_{3} \mathrm{O}_{2} \mathrm{Si}[\mathrm{M}]^{+}: 423.2342$; found: 423.2353.

O-Demethylation of 2-Aminoimidazoles 1d,e; 4-(2-Amino-1methyl-1H-imidazol-4-ylmethyl)phenol (1i); Typical Procedure To a solution of $\mathbf{1 d}(326 \mathrm{mg}, 1.5 \mathrm{mmol})$ in anhyd $\mathrm{CH}_{2} \mathrm{Cl}_{2}(10 \mathrm{~mL})$ was added dropwise a $1 \mathrm{M}$ solution of $\mathrm{BBr}_{3}$ in $\mathrm{CH}_{2} \mathrm{Cl}_{2}(7.5 \mathrm{~mL}$ per methoxy group, $7.5 \mathrm{mmol}, 5$ equiv) at r.t. and the mixture was refluxed for $1 \mathrm{~h}$ at $55^{\circ} \mathrm{C}$. The reaction vessel was cooled in an ice bath and the reaction was quenched by the addition of $6 \mathrm{~N}$ ammonia in $\mathrm{MeOH}(7 \mathrm{~mL})$. After evaporation of the solvent, the residue was subjected to column chromatography $\left(20 \% \mathrm{MeOH}-\mathrm{CH}_{2} \mathrm{Cl}_{2}\right)$ on silica gel basified with ammonia, to afford $167 \mathrm{mg}$ (55\%) of compound 1i; yellow solid; $\mathrm{mp} 114-116^{\circ} \mathrm{C}$.

${ }^{1} \mathrm{H}$ NMR (300 MHz, DMSO- $\left.d_{6}\right): \delta=9.05(\mathrm{br}, 1 \mathrm{H}), 6.99(\mathrm{~d}, J=8.4$ $\mathrm{Hz}, 2 \mathrm{H}), 6.62(\mathrm{~d}, J=8.4 \mathrm{~Hz}, 2 \mathrm{H}), 6.10(\mathrm{~s}, 1 \mathrm{H}), 5.16(\mathrm{~s}, 2 \mathrm{H}), 3.44$ (s, $2 \mathrm{H}), 3.22(\mathrm{~s}, 3 \mathrm{H})$. 
${ }^{13} \mathrm{C}$ NMR $\left(75.5 \mathrm{MHz}, \mathrm{DMSO}-d_{6}\right): \delta=154.9,147.6,135.5,129.8$, 128.9, 114.5, 111.5, 32.9, 30.5.

HRMS-EI: $\mathrm{m} / \mathrm{z}$ calcd for $\mathrm{C}_{11} \mathrm{H}_{13} \mathrm{~N}_{3} \mathrm{O}[\mathrm{M}]^{+}$: 203.1059; found: 203.1059.

\section{4-[2-Amino-1-(4-hydroxybenzyl)-1H-imidazol-4-ylmethyl]phe- nol (1j)}

Prepared from 1e, following the procedure given above; yield: $71 \%$; yellow solid; mp $125-127^{\circ} \mathrm{C}$.

${ }^{1} \mathrm{H}$ NMR (400 MHz, DMSO- $d_{6}$ ): $\delta=9.31(\mathrm{br}, 1 \mathrm{H}), 9.03(\mathrm{br}, 1 \mathrm{H}$ ), $7.01(\mathrm{~d}, J=8.3 \mathrm{~Hz}, 2 \mathrm{H}), 6.98(\mathrm{~d}, J=8.4 \mathrm{~Hz}, 2 \mathrm{H}), 6.69(\mathrm{~d}, J=8.5$ $\mathrm{Hz}, 2 \mathrm{H}), 6.61(\mathrm{~d}, J=8.3 \mathrm{~Hz}, 2 \mathrm{H}), 6.07(\mathrm{~s}, 1 \mathrm{H}), 5.21(\mathrm{~s}, 2 \mathrm{H}), 4.88$ (s, $2 \mathrm{H}), 3.43$ (s, $2 \mathrm{H})$.

${ }^{13} \mathrm{C}$ NMR (100.5 MHz, DMSO- $\left.d_{6}\right): \delta=156.7,155.3,148.5,136.2$, 130.9, $129.5(2 \times), 128.9(2 \times), 128.0,115.2(2 \times), 114.8(2), 110.1$, 46.6, 33.6.

HRMS-EI: $m / z$ calcd for $\mathrm{C}_{17} \mathrm{H}_{17} \mathrm{~N}_{3} \mathrm{O}_{2}[\mathrm{M}]^{+}$: 295.1321; found: 295.1327.

\section{Acknowledgment}

Support was provided by the Research Fund of the University of Leuven. E.V. Van der Eycken thanks the F.W.O. [Fund for Scientific Research - Flanders (Belgium)]. DSE is grateful to the University of Leuven for obtaining a scholarship. EVB thanks the Russian Foundation of Basic Research (RFBR Grant no. 05-03-39022GFEN_a.

\section{References}

(1) (a) Carmely, S.; Kashman, Y. Tetrahedron Lett. 1987, 28, 3003. (b) Carmely, S.; Ilan, M.; Kashman, Y. Tetrahedron 1989, 45, 2193.

(2) To avoid contradictory assignment of the same names to the different isonaamine alkaloids, every isonaamine was named in accordance with the parent isolated isonaamidine, as was originally proposed by Y. Kashman et al. ${ }^{1}$

(3) (a) Akee, R. K.; Carroll, A. R.; Yoshida, W. Y.; Scheuer, P. J.; Stout, T. J.; Clardy, J. J. Org. Chem. 1990, 55, 1944. (b) Chan, G. W.; Mong, S.; Hemling, M. E.; Freyer, A. J.; Offen, P. H.; De Brosse, C. W.; Sarau, H. M.; Westley, J. W. J. Nat. Prod. 1993, 56, 116. (c) Plubrukarn, A.; Smith, D. W.; Cramer, R. E.; Davidson, B. S. J. Nat. Prod. 1997, 60, 712. (d) Fu, X.; Schmitz, F. J.; Tanner, R. S.; Kelly-Borges, M. J. Nat. Prod. 1998, 61, 384.
(4) Molina, P.; Fresneda, P. M.; Sanz, M. A. J. Org. Chem. 1999, 64, 2540.

(5) Alvi, K. A.; Crews, P.; Loughhead, D. G. J. Nat. Prod. 1991, $54,1509$.

(6) Boehm, J. C.; Gleason, J. G.; Pendrak, I.; Sarau, H. M.; Schmidt, D. B.; Foley, J. J.; Kingsbury, W. D. J. Med. Chem. 1993, 36, 3333.

(7) Kawasaki, I.; Taguchi, N.; Yoneda, Y.; Yamashita, M.; Ohta, S. Heterocycles 1996, 43, 1375.

(8) Nakamura, S.; Kawasaki, I.; Yamashita, M.; Ohta, S. Heterocycles 2003, 60, 583.

(9) Alvi, K. A.; Peters, B. M.; Hunter, L. M.; Crews, P. Tetrahedron 1992, 49, 329.

(10) Gross, H.; Kehraus, S.; Koenig, G. M.; Woerheide, G.; Wright, A. D. J. Nat. Prod. 2002, 5, 1190.

(11) Ermolat'ev, D. S.; Babaev, E. V.; Van der Eycken, E. V. Org. Lett. 2006, 8, 5781.

(12) Brown, D. J.; Hoerger, E.; Mason, S. F. J. Chem. Soc. 1955, 4035.

(13) Cherng, Y.-J. Tetrahedron 2002, 58, 887.

(14) Lal, K.; Ghosh, S.; Salomon, R. G. J. Org. Chem. 1987, 52, 1072.

(15) Pedrosa, R.; Andres, C.; Iglesias, J. M. J. Org. Chem. 2001, 66, 243.

(16) (a) Barnett, C. J.; Grubb, L. M. Tetrahedron Lett. 2000, 41, 9741. (b) Aso, K.; Imai, Y.; Yukishige, K.; Ootsu, K.; Akimoto, H. Chem. Pharm. Bull. 2001, 49, 1280.

(17) Grundke, G.; Keese, W.; Rimpler, M. Chem. Ber. 1985, 118 , 4288.

(18) Crystallographic data for 1e reported in this paper have been deposited with the Cambridge Crystallographic Data Centre as supplementary publication No. CCDC 679428. Copies of the data can be obtained, free of charge, on application to CCDC, 12 Union Road, Cambridge CB 2 1EZ, UK [fax: +44(1223)336033 or E-mail: deposit@ ccdc.cam.ac.uk]. Some selected crystallographic data: crystal system, space group: crystal system triclinic; space group $\mathrm{P} \overline{1}$; cell parameters: $a=5.8703(16), b=9.0133(8), c=16.155(2) \AA$; $\alpha=93.778(9), \beta=91.640(10), \gamma=94.461(10)^{\circ} ; \mathrm{V}=849.843$ $\AA^{3} ; Z=2, Z^{\prime}=0 ;$ R-factor $4.9 \%$.

(19) Rybakov, V. B.; Babaev, E. V.; Belykh, E. N. Acta Crystallogr., Sect. E: Struct. Rep. Online 2002, 58, o126.

(20) For analogous methodology to use the readily available pyrimidine-2-one as the masked urea function to prepare 2-aminooxazoles, see: Alifanov, V. L.; Babaev, E. V. Synthesis 2007, 263. 